\title{
Research Progress of the Modified Wood Powder for 3D printing
}

\author{
J.S. Zhang, Y.T. Yang, Z.K. Qin, J.J. Luo \& W. Gao* \\ Forestry College, Guangxi University, Nanning Guangxi 530004, China \\ S.L. Wei \\ Guilin Sen Lin Mei Wooden Furniture Co., Ltd. Guilin Guangxi 541805, China
}

Corresponding author: W. Gao

\begin{abstract}
D printing leads to the changes in manufacturing process, and the research and development of 3D printing supplies is considered as the core competitiveness of this technology. In this paper, it reveals the wood powder modification by establishing performance index of impulse process model to realize the dynamic prediction. The wood powder after grinding can not be directly used for 3D printing, wood powder was modified under high temperature heat treatment in order to make the wood powder with even particle size, dispersed shape and good fluidity, which solve the main problems that wood powder can be employed as printing materials to 3D printing. But there were some disadvantages which would affect the 3D printing performance after the wood powder had been treated, its wettability degrading, sizing saturation reducing, density decreasing and color darkening. After the twice modification, it can obtain the modified parameters and technology by characterizing and analyzing the 7 indexes.
\end{abstract}

KEYWORD: wood powder; 3D printing; high temperature heat treatment; hydrophilic fumed silica; pulse process model

\section{RESEARCH STATUS}

Wood material is manifested by the completely in terms of wood plastic composite, WPC rapid prototyping manufacturing in the research group of Northeast Forestry University (Zhang et al. 2014), draw a lot of performance, Xi'an University of Science and Yang Lai Xia team (Sun 2008, Yang et. al. 2009) combines continuous transition to the theory of color space, This paper present "half color printing" is provided a theoretical basis.

Study abroad is mature. The core technology concentrated in the material developed mainly. 3D printer system is closed by Most commercial, 3D printing consumables for bottleneck to the 3D printing technology competition(Dudek 2013). Overall it can be seen that, supplies are developed significance than equipment, 3D printing has become a national competition of the core technology, forming method, application scope, the levels of 3D printing is decided by material.

Wood powder can not be used directly in 3D printing. The reason is that of the unmodified uneven wood powder particle size, shape, easy to reunite, liquidity is poor, after grinding, the original level, aggregate, condensed matter and flocculation particles all four types of particles had obvious reun- ion phenomenon, during the printing process can not be free fall in 3D printer.

Wood powder after being modified steam temperature heat treatment can be used. It became uniform particle size, shape, dispersion, good liquidity; but there are drawbacks: poor wettability, sizing desaturation, reduced density and darker. Wahab et al. (2013) as an alternative to wood flour and low-cost 3D printing, wood content increases, the size accuracy and surface quality. Populous after high temperature heat treatment, the hydrophilic functional group decrease in absorbance. With the extension of the heat treatment temperature and treatment time gross Masson pine timber. Wood of hydroxyl groups in the infrared absorption spectrum absorption peak intensity is decreased obviously ( $\mathrm{Li}$ et. al. 2009). Fan Min and other (Fan et. al. 2014) heat-treated wood is reduced water absorption. It shows that after the high temperature heat treatment, wood powder of wettability can be decreased. The layer powder, colorless, glue and glue with color realize the interlayer bonding is very unfavorable.

Ingenious modification: High-temperature steam is heat-treated flour have embedded hydrophilic fumed silica, It can make: excellent wettability change, improve the sizing saturation, increased density, lighter color, to make up for the shortcomings of the heat treatment, while improving the li- 
quidity of wood flour. Yang et. al. (2014) 3D printing supplies will be developed into a nano-silica particles are light-cured resin prepared by mixing the ink. Hydrophilic type gas phase silica will be used by reference in this paper.

Domestic and foreign research problems. First of all, is not for wood powder as a 3D printing consumables system research, wood plastic composite material as the material will be involved in wood powder, but the content is low; The second is rare literature for 3D color printing powder were studied.

Then the 3D printing powder modified complex, at the same time make a variety of chemical reagents, the different properties of modified; Finally, no literature systematic study Wooden 3D Powder Modification Mechanism.

\section{INNOVATIONS IN THIS PAPER}

From what has been discussed above, wood powder as $3 \mathrm{D}$ printing consumables will be based on systematic research. The ultimate goal is to wood powder content in more than 95\%, other ingredients into a single silicon dioxide. Suitable for 3D printing, keep the good performance of wood powder. "Half the color print" be studied at the same time. Although can't achieve full color. And according to the modified powder characteristics, reveal the mechanism of modification, impulse process model is established, when a performance change of wood powder, predict other evolution and trend of performance index and the quality of the product.

\section{MAIN CONTENTS}

\subsection{Modified}

High temperature heat treatment modification of wood powder and water type gas phase silica embedding modification.

The wood is ground into flour, 240 mesh sorting, Modified high temperature treatment, wood powder is 300 mesh sieve separation.

To explore the high temperature steam heat modification of wood powder technology: Under the water protection. After different heat treatment temperature, processing time and wood powder particle size, shape, reunion, liquidity, wettability, density, porosity, saturation and color changes, find out the optimum heat treatment process, get fit 3D printing pavement and half color forming mechanism.

With embedded modified hydrophilic fumed silica: The negative impact of high temperature heat treatment of wood flour is used as modified hydrophilic fumed silica. Negative influence mainly has: low density, wettability can reduce, color deepened. Wood powder density decreased, the mechanical properties of the 3D product is reduced. Wettability can be reduced, is not conducive to layer glue. Color deepens. It is not conducive to the expression of color 3D printing products. Therefore will argue the embedding different proportions of hydrophilic type gas phase silica on the effects of secondary modified wood powder.

Hydrophilic fumed silica bulk density up to 2.2 $\mathrm{g} / \mathrm{cm}^{3}$, much higher than the bulk density of wood powder, can effectively improve the density of layer. Hydrophilic fumed silica is also one of excellent superfine inorganic materials, because of its small particle size. For this specific surface area, surface energy, surface adsorption capacity is strong, the wettability of sizing problems in 3D printing was encased wood powder are improved, and Make up for the wettability of wood powder in the process of high temperature heat treatment can reduce the problem. Hydrophilic type gas phase silica is pure white. Mixed in the heat treatment of wood, the brightness of the wood powder are added. Can be improved due to the high temperature heat treatment after the color too dark. At the same time. Hydrophilic fumed silica having a micro-ball effect. The friction between the particles can be mixed with wood powder, to improve the effect of powder liquidity.

\subsection{Characterization research}

Analysis 7 flour modification process performance metrics change, exploring the Interaction Factors.

\subsubsection{Particle}

Analysis particle size matching law, clear the relationship between particle size and other performance indicators

If the wood flour particles are small, sizing bond forming process is easy, but easy to form flocculation, occurs powder agglomeration, easy to stick to the roller surface, resulting in difficult to form a uniform thin layer; if wood flour is larger particles, in the sizing process is not easy penetration while its smaller surface area, and other wood flour particles are bonded difficult affect the mechanical properties of the inner layer.

This paper does not require a consistent particle size, a variety of powder will be able to match the size in rules. For fibrous mainly to flour, the size of the particles matches the geometric model before and after modification (Fig. 1).

(a) untreated

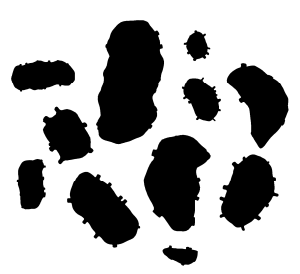

(b) treated

Fig. 1 wooden powder particles matching geometry 
As can be seen from the model, after the heat treatment, surrounding from wood flour "fluff" and very fine particles are reduce or disappeared. In the process of dusting difficult to form floc, promote smooth roll surface and improve the uniformity of a thin layer.

On this basis, determine the impact of the relationship between particle size and agglomeration, flowability, wettability, sizing saturation.

\subsubsection{Reunion}

Analysis the relationship of particle morphology, particle size and argumentation reunion, color and fluidity.

Analyzed wood flour particles shapes under different parameters treatment in order to avoid particle agglomeration. According to the outline of the border flour particle shape or image of each point, the particles can be divided into four types: Primary level, the aggregates, condensates and flocculation particle type, as shown in Fig. 2. One of the purpose after high temperature heat treatment of wood flour is to avoid significant agglomeration, as much as possible to improve the proportion of primary-particles.

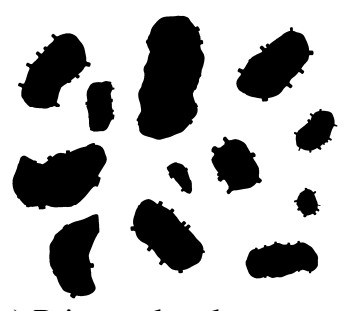

(a) Primary level

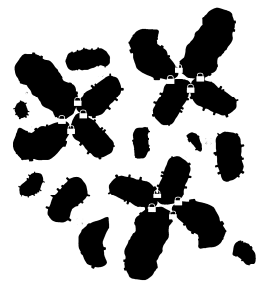

(c) Condensates

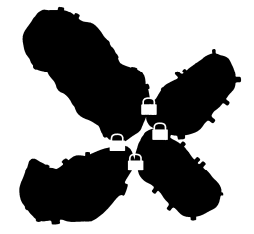

(b) The aggregates

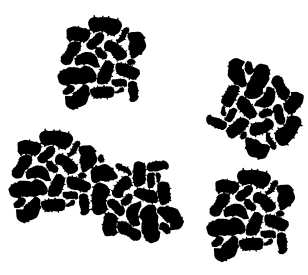

(d) Flocculation
Fig. 2 Flour reunion shape model figure

\subsubsection{Liquidity}

Analysis the flow rate, access to adapt to free fall powder

Unmodified flour illiquid, when performance measurement, in addition to the flow meter conduit powder can fall outside, other wood flour can not fall freely in the flow meter funnel, forming arch shape, and they can only go through by shaken. After high temperature treatment, they can meet the liquidity requirements of dusting basically. In order to improve its performance further, embedding hydrophilic fumed silica, to play its micro-ball effect, reducing the coefficient of friction between the wood flour particles, analysis flow properties when the amount of wood flour is different.

\subsubsection{Porosity}

Binding density, analysis of porosity impact on wetting, saturation, color

Research on wood flour change after heat treatment, hydrophilic fumed silica embedded sizing density. To measure true density and bulk density of wood flour, porosity was calculated on this basis.

Study on the porosity of the interlayer sizing permeability. The greater the porosity of wood flour, glue will penetrate in the surface sooner, but 3D printing density of the product influenced seriously by the glue, at the same time the accuracy of the product surface is reduced, the porosity of the flour needs to be reasonable control

\subsubsection{Wettability}

Based on the powder surface tension, analysis wetting properties, obtained modification process. In the process of laminates wood flour, we hope glue surface wettability strong enough to penetrate quickly between the particles to increase uniformly cured to form a fine texture of $3 \mathrm{D}$ printing products. In this thesis, after high temperature heat treatment, wood flour wetting properties decrease, therefore we use hydrophilic fumed silica entrap modified. Course of the study, we will track the impact of different amount of modified substances added on the wetting properties of wood flour, make a comparative study combine with other properties of wood floor.

\subsubsection{Sizing saturation}

With the porosity, Analysis saturation type. Sizing saturation refers when 3D printing, after each dusting sizing, glue and wood flour pore volume occupied by the volume ratio. When printing molding process, wood glue is moistened, and with the glue penetration, the chemical and physical reactions take place in the flour particles, they form a solid bridge, so as to achieve adhesion between the wood flour particles. The capillary filling is shown in Fig. 3 is the most ideal.
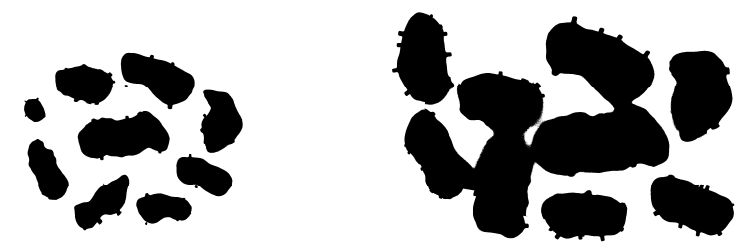

(a) Slushy

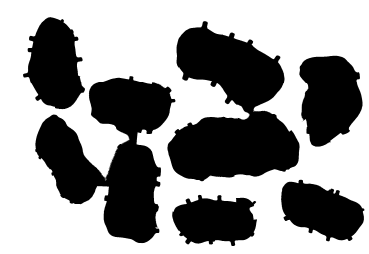

(c) Ribbon cable (b) Capillary

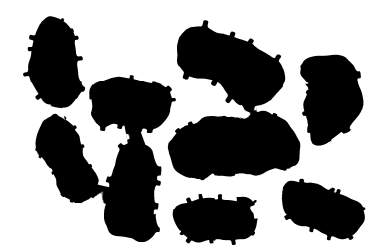

(d) Pendularly
Fig. 3 Filled with wood flour glue schematic 


\subsubsection{Color}

Based on CMYK theory, achieve "semi-color printing." In the course of this study, 3D System companies supporting VisiJet PXL series glue is used by us, the series glue are five types: CMYK and transparent glue, may be formed RGB color model, achieve so-called "full-color printing."

However, the present paper carrier is wood flour, different from white paper, after high temperature treatment modification, Red-Green axis flour color chromaticity index are rose, Yellow-Blue axis chromaticity index are decreased, the overall body color becomes darker, effected by wood-flours color, it can not achieve "full-color printing." For example, the existing wood flour colors almost is yellow, only print yellow $(\mathrm{Y})$, red $(\mathrm{R})$, black $(\mathrm{K})$, green $(\mathrm{G})$ and other regional color products, Can not print cyan $(\mathrm{C})$, magenta (M), and blue (B) color product, so this paper is called "semi-color printing."

Therefore, this paper will focus on the analysis of different heat treatment flour modified parameters and different hydrophilic fumed silica modified embedded situation, using the CMYK color card, looking for the model which color printing can be achieved, feedback to the model build.

Model building color, when printing, head feeling the gap between detection and flour color, calculate the difference automatically, mobilizing CMYK four colors to compensate.

As can be seen, the seven complex factors influence each other, when one factor changes, along with changes in other performance indicators, is difficult to have a fixed heat treatment and embedded modified parameters and process. With different species, different parts of heartwood and sapwood, different strains drawn, the above properties of wood flour also changes.

\subsection{Key problems to be solved}

The key technology: determining the steam temperature heat treatment of wood flour and embedding hydrophilic fumed silica modified to ensure the wood flour adapts for 3D printing.

Critical theory: establish impulse process model from the intricate performance indexes. Predicting changes in other performance indexes, the quality of the product which caused by partial change.

\section{RESEARCH SIGNIFICANCE}

3D printing wooden supplies was developed. Access to get new types of supplies. Environmentally friendly features wood is reflected perfectly. 3D printing modification of wood powder and forming mechanism was exploring. Impulse process model was constructed. For wood powder changes by a certain performance index. Evolution and trends of oth- er performance indicators is predicted, improve the modification process parameters.

\section{ACKNOWLEDGEMENTS}

The authors are grateful for the support of the Funds of Guangxi Scientific Research and Technological Development Program of China (Grant No. GKN 14121007-6-1) and the Innovation \& entrepreneurship training program for college students in Guangxi University (Grant No. 201410593200).

\section{REFERENCES}

DUDEK, P. 2013. Fdm 3D printing technology in manufacturing composite elements[J]. Archives of Metallurgy and Materials, 58(4): 1415-1418.

FAN M., NI O.Q., YU Z.Q., ZAHNG K.M., LV Z.K. 2014. Effect of thermal treatment on the physical and mechanics properties of Dalbergia melanoxylon wood. Journal of Nanjing Forestry University (Natural Sciences Edition), (02):3135.

LI X.J., LIU Y., GAO J.M., WU Y.Q., YI S.L., WUZ.P.. 2009. FTIR and XRD for wood with high-temperature heating treatment. Journal of Beijing Forestry University, (31): 104107.

SUN G.G.. (2008) Research of Materials in 3DP Manufacturing Technology. Xi'an University of Science and Technology: $12-15$

WAHAB, S., WAGIMAN, A., IBRAHIM, M. (2013) Development of wood-based composites material for 3D printing process. In 3rd International Conference on Mechanical and Manufacturing Engineering, ICME 2012, November 20, 2012 - November 21, 2012. Malaysia, Trans Tech Publications Ltd. pp 987-991

YANG L.X., CHI X.F., ZHANG N.F.. 2009. Interpolation method of color gradually changed on 3-D printing technology. Journal of Xi'an University of Science and Technology, (29): 214-218.

YANG, H., SHARON, N. M. L., QI, X.,WEI, J. (2014) Preliminary study on nano particle/photopolymer hybrid for 3d inkjet printing, 164-169. 